\title{
Clostridium difficile, sulphasalazine, and ulcerative colitis
}

\author{
D.A. Burke and A.T.R. Axon \\ Gastroenterology Unit, The General Infirmary, Leeds, LS1 3EX, UK.
}

\begin{abstract}
Summary: Clostridium difficile has been implicated in the relapse of ulcerative colitis. Controversy exists over this role and its relationship to sulphasalazine exposure. Sixty two of 77 patients with a documented relapse of ulcerative colitis were investigated for the presence of Clostridium difficile, or its toxin, prior to hospitalization. There was a low incidence of detection which was related to antibiotic exposure (2/62). Sampling during the treatment period showed that the occurrence of Clostridium difficile in the stool was related to antibiotic treatment (2/66). Fifty six percent of patients were taking sulphasalazine, none of whom became culture or toxin positive. This study demonstrates that Clostridium difficile is not related to relapse of ulcerative colitis and is not secondarily acquired during relapse unless the patient is exposed to antibiotics. Sulphasalazine does not predispose to acquisition of Clostridium difficile. There is no role for routine screening or treatment of Clostridium difficile in ulcerative colitis.
\end{abstract}

\section{Introduction}

The role of Clostridium difficile in inflammatory bowel disease and its relationship to sulphasalazine exposure is controversial. Studies from different parts of the world have suggested that it may cause relapse $\mathrm{e}^{1,2,3}$ whilst others have disputed this. ${ }^{4,5,6}$ Sulphasalazine has been proposed as a predisposing factor ${ }^{7}$ and a protective factor. ${ }^{8}$ Variations in sampling, timing of collection, absence of either culture or toxin results, and population differences may contribute to some of the discrepancies in these studies. Detection of the organism or its toxin during a relapse of inflammatory bowel disease cannot necessarily implicate it in the causation of the relapse without taking into account the temporal relationship of its appearance during the attack in order that secondary acquisition can be excluded. Alternatively, changes in the faecal ecology secondary to the disease process may facilitate detection of the organism in inflammatory bowel disease.

Because of the diagnostic and therapeutic implications of this organism having a role in inflammatory bowel disease this study was undertaken to investigate Clostridium difficile in patients presenting with a relapse of ulcerative colitis.

\section{Patients and methods}

Stool samples were obtained from patients with an acute attack of ulcerative colitis attending the inflammatory bowel disease clinic. The diagnosis was established by endoscopic and histological criteria. Stool was

Correspondence: D.A. Burke M.B., B.S., M.R.C.P. Accepted: 6 May 1987 collected into sterile universal containers, immediately dispatched to the laboratory, and maintained at $4^{\circ} \mathrm{C}$ during analysis. Stools were collected in the outpatient department on presentation in all outpatients and most inpatients; where this was not possible samples were obtained within 12 hours using disposable containers to eliminate contamination.

Seventy seven patients with an acute relapse of ulcerative colitis were studied during the period October 1984 to March 1986 (42 males, 35 females) age range 19-75 (mean 42.6). Twenty patients had disease limited to the rectum, in 27 the disease involved the left side of the colon, and 30 patients had disease extending beyond the splenic flexure. Nine patients had severe disease, 49 moderate, and 19 mild (Truelove and Witts criteria). ${ }^{9}$ The length of history of the attack ranged from 3 days to 52 weeks (median 6 weeks). Thirty five patients were actively taking sulphasalazine, another 27 had received it at some time in the past, and 5 were receiving 5-amino salicylic acid.

Clostridium difficile was isolated under anaerobic conditions using CCFA Agar (Cefoxitin, Cycloserine, Fructose Agar). Toxin was detected by examining stool supernatant, for a cytopathic effect on HeLa cells which could be neutralized by Clostridium sordellii antitoxin. In addition stool was routinely cultured for salmonella, shigella and campylobacter species.

\section{Results}

Sixty two patients were able to supply a suitable stool sample prior to commencing treatment. Only 1 patient had a positive culture for Clostridium difficile but had 
no detectable stool toxin, he had been treated with cotrimoxazole for a chest infection and his symptoms resolved when the antibiotic was withdrawn. Another patient was found to be culture negative, but toxin was detected at very low titres $(1: 2)$, there had been no history of antibiotic exposure in this case. Only 4 patients had received antibiotics 1-2 months before the onset of their symptoms, and only 1 of these was culture positive.

There were 9 patients in whom no initial stool sample was obtained, but who provided a specimen during the course of treatment. All of these were negative for culture and toxin. Stools were assayed in a further 57 patients during treatment. Two cases were culture positive for Clostridium difficile but no toxin was detected. Both had received antibiotics during the treatment period, 1 patient in hospital the other as an outpatient. The inpatient was given vancomycin $125 \mathrm{mg}$ t.d.s. orally on the basis of the positive culture result despite the fact that recovery from his colitis did not show any relapse in relation to antibiotic treatment or positive stool culture result. Three inpatients who required emergency colectomy were all Clostridium difficile negative at presentation. Six patients did not have Clostridium difficile culture or toxin assay before or during treatment but they all responded to treatment appropriate for their colitis. In no case was any other bacterial pathogen isolated before or during treatment.

\section{Discussion}

Reports in 1980 of an association with relapse of inflammatory bowel disease and the presence of Clostridium difficile or its toxin in the stool led to the suggestion that it might have a role in the relapse of IBD. ${ }^{1,2}$ Further studies have both supported ${ }^{3}$ and refuted ${ }^{4,5,6}$ this view. Explanations put forward for the disparity in these reports have included population differences in patient groups, non-specificity of the toxin assays used, together with the absence of supportive culture results. The timing of cultures in relation to presentation and hospital admission has not been adequately documented and, because of this, stool Clostridium difficile positivity as a consequence of secondary acquisition during a period of active inflammation could not be excluded. Cultures obtained from hospitalized patients may represent nosocomial spread..$^{10,11}$

In this study we have eliminated some of these problems by studying a group of patients whose diagnosis had been sigmoidoscopically and histologically substantiated, and by collecting samples before the patient was admitted to the hospital environment to eliminate nosocomial contamination.
Furthermore, both culture and toxin assay were undertaken before and during treatment to detect any secondary colonization with Clostridium difficile.

Our results show that Clostridium difficile is not associated with relapse of ulcerative colitis and furthermore it is not acquired during the period of active inflammation even in those patients in hospital where during the study period there were sporadic cases and even clusters of Clostridium difficile-associated diarrhoea in other patient groups throughout the hospital, indicating that the organism was probably in the environment. Barrier nursing is not normally employed for our colitic patients and they are managed on general medical and surgical wards throughout the hospital. Very few patients had been exposed to antibiotics, other than sulphasalazine. Only 1 patient appeared to have symptoms in association with a positive stool culture and in this case there was an identifiable antibiotic aetiology.

The fact that $56 \%$ of our patients were taking sulphasalazine and none of them became culture positive does not support the view that sulphasalazine predisposes to the development of Clostridium difficile associated disease. In vitro studies ${ }^{12}$ demonstrating the inhibitory effect of sulphasalazine, sulphapyridine and to some extent 5-amino salicylic acid on Clostridium difficile also refute the suggestion of Greenfield et al. ${ }^{\circ}$

The cost of culture and toxin assay is approximatel $£ 5.00$ per sample in a laboratory with establishes. tissue culture facilities, and the cost of treatment fo $F$ five days with vancomycin $125 \mathrm{mg}$ t.d.s/day is about $£ 45.00$. Consequently it would require considerable expenditure in time, and money to undertake either routine diagnosis or treatment of Clostridium difficile in patients with ulcerative colitis. Furthermore toxin assay is not readily available in all centres.

Patients with inflammatory bowel disease may respond to treatment with vancomycin. ${ }^{\prime}$ This observation would appear to add support to the theory that Clostridium difficile is involved in relapse, but it does not follow that the improvement is necessarily related to its activity against Clostridium difficile. A recent study from Dickinson et al. ${ }^{13}$ showed that there was a trend towards improvement in those treated with vancomycin but that it was not related to eradication of Clostridium difficile. It is therefore possible that its benefit is not related to its antibiotic potential, but some other property, just as metronidazole has been shown to be of benefit in Crohn's disease, ${ }^{14}$ possibly due to its in vitro immunosuppressive activity. ${ }^{15}$

This study shows that Clostridium difficile is not involved in relapse of idiopathic ulcerative colitis, and that consequently there is no need to screen for Clostridium difficile unless there is a history of antibiotic exposure. Patients with ulcerative colitis may develop infection with Clostridium difficile following antibiotic exposure as any other patient group. 


\section{References}

1. LaMont, J.T. \& Trnka, Y.M. Therapeutic implications of Clostridium difficile toxin during relapse of chronic inflammatory bowel disease. Lancet 1980, i: 381-383.

2. Bolton, R.P., Sherriff, R.J. \& Read, A.E. Clostridium difficile associated diarrhoea: a role in inflammatory bowel disease. Lancet 1980 , i: 383-384.

3. Trnka, Y.M. \& LaMont, J.T. Association of Clostridium difficile toxin with symptomatic relapse of chronic inflammatory bowel disease. Gastroenterology 1981, 80: 693-696.

4. Meyers, S., Mayer, L., Bottone, E., Desmond, E. \& Janowitz, H.D. Occurrence of Clostridium difficile toxin during the course of inflammatory bowel disease. Gastroenterology 1981, 80: 697-700.

5. Keighley, M.R.B., Youngs, D., Johnson, M., Allen, R.N. \& Burdon, D.W. Clostridium difficile toxin in acute diarrhoea complicating inflammatory bowel disease. Gut 1982, 23: 410-414.

6. Rolny, P., Jarnerot, G. \& Mollby, R. Occurrence of Clostridium difficile toxin in inflammatory bowel disease. Scand J Gastroenterol 1983, 18: 61-64.

7. Greenfield, C., Aguilar Ramirez, J.R., Pounder, R.E. et al. Clostridium difficile and inflammatory bowel disease. Gut 1983, 24: 713-717.

8. Tremaine, W.J., Billie, J., Huizenga, K.A., Washington, J.A. \& Ilstrup, D.M. Factors which influence the occurrence of Clostridium difficile infections in inflam- matory bowel disease. Gastroenterology 1983, 84: 1337.

9. Truelove, S.C. \& Witts, L.J. Cortisone in ulcerative colitis. Final report on a therapeutic trial. Br Med J 1955, 2: $1041-1048$.

10. Greenfield, C., Burroughs, A., Szathowski, M. et al. Is pseudomembranous colitis infectious? Lancet 1981, i: 371-372.

11. Kim, K.H., Fekety, R., Batts, D.H. et al. Isolation of Clostridium difficile from the environment and contacts of patients with antibiotic associated colitis. J Infect Dis 1981, 143: 42-49.

12. Sandberg-Gertzen, H., Kjellander, J., Sundberg-Gilla, B. \& Jarnerot, G. In vitro effects of sulphasalazine, azodisal sodium, and their metabolites on Clostridium difficile and some other faecal bacteria. Scand J Gastroenterol 1985, 20: $607-612$.

13. Dickinson, R.J., O'Conner, H.J., Pinder, I., Hamilton, I., Johnston, D. \& Axon, A.T.R. Double blind controlled trial of oral vancomycin as adjunctive treatment in acute exacerbations of idiopathic colitis. Gut 1985, 26: 1380-1384.

14. Bernstein, L.H., Frank, M.S., Brandt, L.J. \& Boley, S.J. Healing of perineal Crohn's disease with metronidazole. Gastroenterology 1980, 79: 357-365.

15. Grove, D.I., Mahmoud, A.A.F. \& Warren, K.S. Suppression of cell-mediated immunity by metronidazole. Int Arch Allergy Appl Immunol 1977, 54: 422-427. 